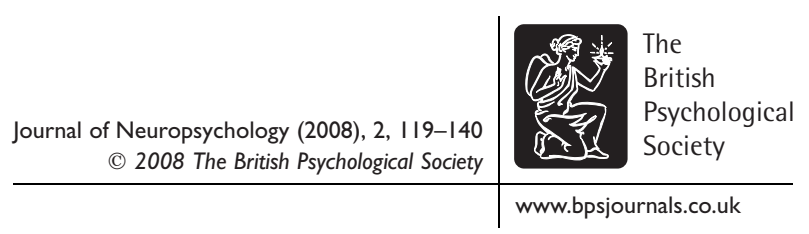

\title{
Face detection in normal and prosopagnosic individuals
}

\author{
Lúcia Garrido'*, Brad Duchaine' and Ken Nakayama² \\ 'Institute of Cognitive Neuroscience, Department of Psychology, University College \\ London, London, UK \\ ${ }^{2}$ Vision Sciences Laboratory, Department of Psychology, Harvard University, \\ Cambridge, USA
}

\begin{abstract}
Face detection, the process of finding a face in a visual scene, is a critical step in face processing, yet it has received relatively little attention compared with other face processes. The present study addresses this crucial first stage by investigating the effect of inversion on face detection and by examining how individuals with developmental prosopagnosia perform on face detection tasks. Fourteen control participants and fourteen individuals with developmental prosopagnosia (DPs) were tested with two face detection tasks: (I) Face versus Non-Face, where arrays of small images were presented, one of which could contain a face and (2) Face versus Face Parts, where a two-tone face could be embedded in a larger array of similar two-tone face parts. On each trial, participants made a speeded response if a face was present in the visual display. On almost all measures both normal and prosopagnosic individuals showed strong inversion effects with significantly worse performance with inverted faces. This shows that the simple task of detection can show inversion effects comparable to those seen for other face tasks, including recognition. Finally, while there were prosopagnosics who were well within the normal range for detection, there were significant group differences, particularly for the case of the Face versus Face Parts, where prosopagnosics were worse than controls on upright but not on inverted face detection.
\end{abstract}

Face detection is the process of finding a face in a visual scene. It is considered a crucial stage in machine vision research and computer scientists have devoted considerable effort to building systems that automatically detect faces in images (Viola \& Jones, 2004; Yang, Kriegman, \& Ahuja, 2002). However, surprisingly little research has examined human face detection. Even though detecting a face would appear to be an essential prerequisite for the activation of face-specific processes, the most influential models of face recognition have only addressed the cognitive and neural mechanisms used to

* Correspondence should be addressed to Lúcia Garrido, Department of Psychology, Institute of Cognitive Neuroscience,
University College London, Alexandra House, I 7 Queen Square, London WCIN 3AR, UK (e-mail: m.garrido@ucl.ac.uk). 
process faces after they have been identified as faces (Bruce \& Young, 1986; Burton, Bruce, \& Hancock, 1999; Haxby, Hoffman, \& Gobbini, 2000). The present study attempts to contribute to the understanding of face detection by investigating the effects of inversion on face detection tasks, and by testing individuals with face processing impairments on those tasks.

\section{Inversion effects in face detection}

For a long time, it has been known that the inversion of faces causes a substantial drop in the performance of tasks that require the discrimination of two faces (e.g. Freire, Lee, \& Symons, 2000; Riesenhuber, Jarudi, Gilad, \& Sinha, 2004; Yovel \& Kanwisher, 2004) or the recognition of previously seen faces (e.g. Diamond \& Carey, 1986; Duchaine \& Nakayama, 2006; Moscovitch \& Moscovitch, 2000; Yin, 1969). These large inversion effects are considered a hallmark of face processing, because inversion of other visual stimuli has a much smaller effect on performance (Diamond \& Carey, 1986; Farah, Tanaka, \& Drain, 1995; McKone, Kanwisher, \& Duchaine, 2007; Yin, 1969). Because it remains unclear whether face detection also elicits large inversion effects, the first aim of this study is to investigate whether face detection is similarly affected by inversion.

A number of studies have investigated face inversion in face detection paradigms. For example, Purcell and Stewart (1986, 1988), studying what they called the facedetection effect, asked participants to detect the presence of a face when it was presented very briefly and followed by a mask. Typically, participants were asked to say whether the stimulus was to the left or to the right of the fixation point. They found that participants were more accurate in determining the location of the face and they were able to perform accurately with shorter presentation times when the stimulus was a normally oriented face when compared with an inverted face. In addition, large inversion effects have been found in face categorization tasks using Mooney stimuli (Andrews \& Schluppeck, 2004; George, Jemel, Fiori, \& Renault, 1997; George, Jemel, Fiori, Chaby, \& Renault, 2005; Kanwisher, Tong, \& Nakayama, 1998). However, other studies, using more ecologically valid paradigms, have found small inversion effects in face detection (Lewis \& Edmonds, 2003, 2005; Rousselet, Mace, \& Fabre-Thorpe, 2003; VanRullen, 2006). For example, in a study by Lewis and Edmonds (2003), visual scenes were taken from a soap opera. The visual displays were tessellated into nine rectangular cells, one of which could contain a face. Participants were required to judge as quickly as possible whether or not a stimulus contained a face. The authors carried out a number of manipulations and found that inverting, blurring, reducing the contrast of the images, reversing the pattern of luminance, or reversing the pattern of hue all led to slower face detection. However, the effect of inversion was very small (on average, less than $20 \mathrm{~ms}$ ). In another study that also used face detection in natural scenes, inversion was again found to cause little impairment on performance (Rousselet et al., 2003). Similarly, two studies using visual search tasks found that the reaction times needed to detect a face among distractors were only slightly slower for inverted stimuli, and were not affected by the number of distractors for both upright and inverted faces (Lewis \& Edmonds, 2005; VanRullen, 2006).

Lewis and Edmonds $(2003,2005)$ suggested that these small inversion effects show that face detection is not affected by inversion as much as face recognition. They considered their results to be an evidence for separate detection and recognition 
mechanisms in face processing. It is possible, however, that the small inversion effects seen in these studies might result from the type of stimuli used, and that detection per se could show inversion effects comparable to those found for other face processes. In more naturalistic tasks, there are a number of other cues that are available for both upright and inverted faces which might lead to a good performance, such as the presence of bodies, skin colour, and contextual cues. We expect that different stimuli that force reliance more on the structure of the face could produce larger inversion effects, and our tasks explore this possibility.

\section{Face detection in developmental prosopagnosia}

The second aim of this study is to investigate whether individuals with severe face recognition impairments (prosopagnosia) can detect faces normally. Some studies with patients with prosopagnosia following brain damage (acquired prosopagnosia) have shown preserved face detection (e.g. Bruyer et al., 1983; de Gelder \& Rouw, 2000; Rossion et al., 2003; Schiltz et al., 2006), and studies with individuals with developmental prosopagnosia have also found that they performed well on face detection tasks (de Gelder \& Rouw, 2000; Duchaine, Nieminen-von, New, \& Kulomaki, 2003; Duchaine, Yovel, Butterworth, \& Nakayama, 2006; Le Grand et al., 2006). These results have been interpreted as evidence that face detection can be dissociated from face recognition processes.

Most of these studies, however, used categorization tasks in which participants decided whether a centrally presented stimulus was a face or a scrambled face in some tasks, or a face or an object in other cases. Lewis and Ellis (2003) pointed out that these tasks do not closely simulate face detection in everyday life in that faces are presented alone without any distractors or context. Moreover, when asked to decide whether a stimulus is a face or a scrambled face, participants, rather than detecting a face, simply need to make a judgment about the relative positions of facial features. We think that the study of face detection in prosopagnosia would benefit from the adoption of paradigms other than the face classification ones (for a review of face detection paradigms, please see Lewis \& Ellis, 2003). Our tasks are designed to be more ecologically valid in that the faces are surrounded by distractors. As visual search type tasks, they measure how well individuals can find a face in a visual display that contains other elements. These demands are also much more akin to the task demands placed on machine vision systems carrying out face detection.

To summarize, in the present study, we employed two face detection tasks in which participants had to find a face in a visual array. We attempted to use stimuli that were highly reliant on the structure of the face, and to use an ecologically valid paradigm in which faces were embedded in arrays that contained other elements. The first task required that participants detect a face within an array (Figure 1), a method similar to that used by Lewis and Edmonds (2003) but removing context cues and adding noise to the pictures. The second task required detection of a face configuration among a set of distractors containing face parts (Figure 2), thus increasing the chances that face detection mechanisms reliant on face configurations would be required. In both the cases, participants had to decide whether a face was present in the stimulus. We used these tasks to investigate how the inversion of faces affects their detection, and also to explore how well individuals with developmental prosopagnosia can detect a face in a visual array. 


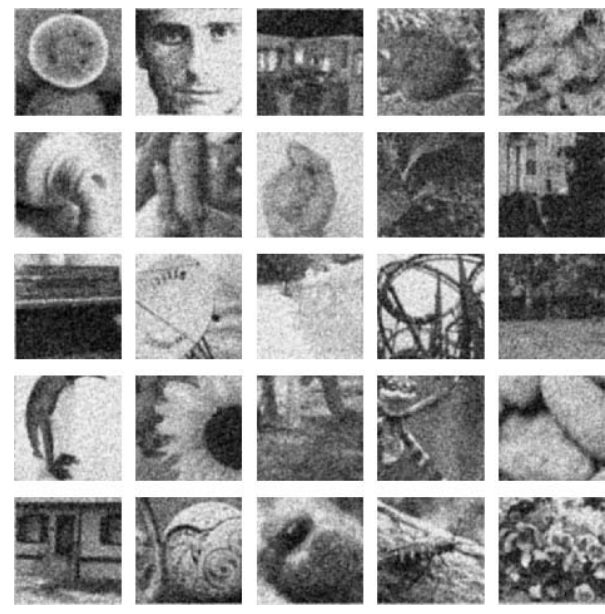

Upright
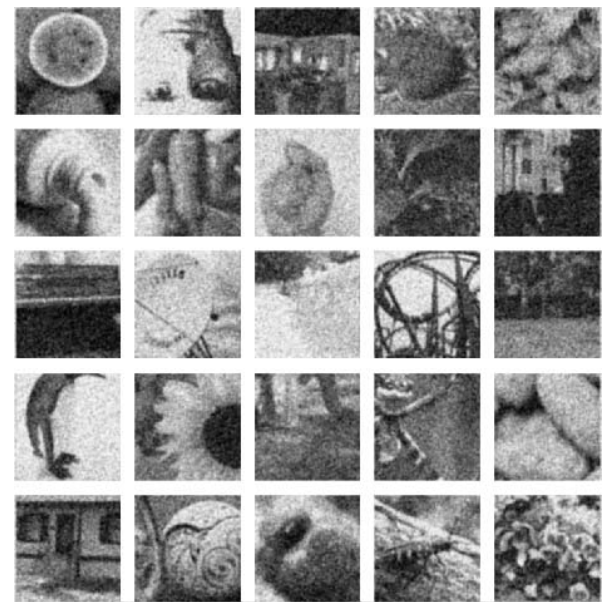

Inverted

Figure I. Example of stimuli used in the Face versus Non-Face task. The figure on the left contains an upright face and the figure on the right contains an inverted face.

\section{Method}

\section{Participants}

Control participants

Fourteen control participants were tested (ten females). They ranged between 20 and 39 years of age and the mean age was 28.1 years $(S D=6.7)$. All of them reported normal or corrected to normal vision and did not report any history of neurological damage.

Developmental prosopagnosics (DPs)

Fourteen DPs were tested (ten females). They ranged between 19 and 39 years of age and the mean age was $30.8(S D=5.4)$. They contacted us through our research website

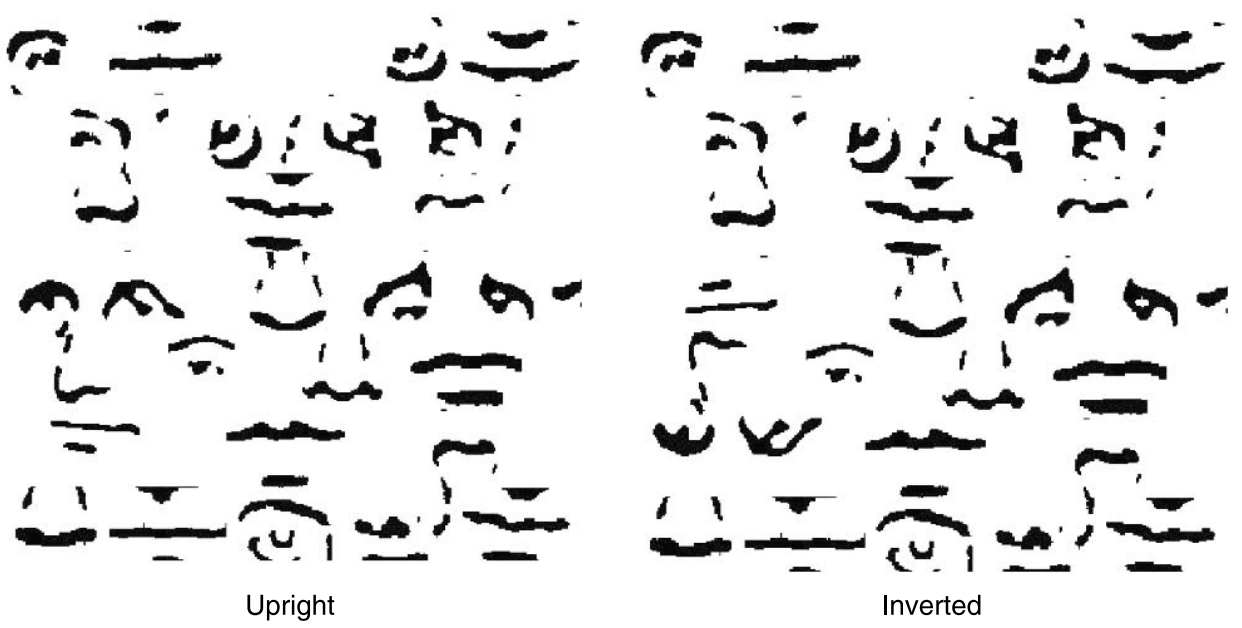

Figure 2. Example of stimuli used in the Face versus Face Parts task. The figure on the left contains an upright face and the figure on the right contains an inverted face. 
(http://www.faceblind.org). All complained of lifelong severe problems recognizing faces and reported considerable difficulties recognizing relatives, friends, or co-workers in everyday life. Table 1 shows their identification codes, ages and occupations. None of them was aware of any incident that could have caused brain damage, and all reported normal or corrected to normal visual acuity. F30a, F35, F39b and M34 are siblings and some of their scores were described in Duchaine, Germine, and Nakayama (2007). To test low-level perceptual abilities, all DPs except F19 and M34 performed four tests (Length Match, Size Match, Orientation Match and Position of Gap) of the Birmingham Object Recognition Test (BORB) (Riddoch \& Humphreys, 1993). (See Appendix A for individual results). All DPs tested performed within the normal range on the BORB tests.

Table I. Age and occupation of each DP

\begin{tabular}{lll}
\hline Code & Age & \multicolumn{1}{c}{ Occupation } \\
\hline F19 & 19 & Model \\
F24 & 24 & Civil servant \\
F26 & 26 & PhD student \\
F30a & 30 & Master student counselling \\
F30b & 30 & Civil servant \\
F32a & 32 & Database manager \\
F32b & 32 & Software developer \\
F35 & 35 & University administrator \\
F39a & 39 & Artist \\
F39b & 39 & Electrical engineer \\
M29 & 29 & Software developer \\
M30 & 30 & Designer \\
M32 & 32 & Researcher \\
M34 & 34 & Programmer \\
\hline
\end{tabular}

To establish that the self-reported DPs actually have problems with face recognition, we assessed them with two tests involving recognition of facial identity: the Cambridge Face Memory Test (CFMT) (Duchaine \& Nakayama, 2006) and the Famous Faces Test (Duchaine \& Nakayama, 2005). Table 2 shows that all 14 DP individuals had impaired scores on both tests of face recognition. Table 2 also shows their performance on the Cambridge Face Perception Test (CFPT) (Duchaine, Germine et al., 2007; Duchaine, Yovel, \& Nakayama, 2007). The CFPT is designed to minimize memory demands to test the perception of faces. Not all DPs showed impairments on this task, but we included it to display how impairments on this perceptual task relate to impairments on face detection tasks. In addition, all DPs were tested on within-category object recognition tests and results can be seen in Appendix B. These scores allow us to investigate whether face detection performance is related to object recognition impairments. These tests are described below. Each DP's result for each test was individually compared with those of control participants using the modified $t$ test devised by Crawford and Howell (1998) for use with single cases.

Cambridge face memory test

The CFMT (Duchaine \& Nakayama, 2006) has three stages. In the introduction, participants saw three different pictures of six different males who they were required 
Table 2. DPs results on tests of face processing

\begin{tabular}{|c|c|c|c|c|c|}
\hline \multirow[b]{2}{*}{ Code } & \multirow[b]{2}{*}{ CFMT } & \multicolumn{2}{|c|}{ Famous faces } & \multicolumn{2}{|c|}{ CFPT } \\
\hline & & Identified & Exposed & Upright & Inverted \\
\hline F19 & $32 *$ & $13 *$ & 52 & 34 & 66 \\
\hline F24 & $38 *$ & $9 *$ & 56 & $66 *$ & $86 *$ \\
\hline F26 & $36 *$ & $19 *$ & 57 & 50 & $90 *$ \\
\hline $\mathrm{F} 30 \mathrm{a}$ & $40 *$ & $15^{*}$ & 41 & 60 & 66 \\
\hline F30b & $37 *$ & $13 *$ & 51 & $82 *$ & $90 *$ \\
\hline $\mathrm{F} 32 \mathrm{a}$ & $43^{*}$ & $36^{*}$ & 56 & 52 & 74 \\
\hline $\mathrm{F} 32 \mathrm{~b}$ & $42 *$ & $28^{*}$ & 52 & $74 *$ & $90 *$ \\
\hline F35 & $31 *$ & $14 *$ & 53 & $68^{*}$ & 60 \\
\hline $\mathrm{F} 39 \mathrm{a}$ & $4 I^{*}$ & $31 *$ & 58 & 40 & 56 \\
\hline F39b & $39 *$ & $6 *$ & 47 & $66^{*}$ & $88^{*}$ \\
\hline M29 & $35^{*}$ & $21 *$ & 55 & 50 & 74 \\
\hline M30 & $42 *$ & $28^{*}$ & 60 & $64^{*}$ & 44 \\
\hline M32 & $40 *$ & $20 *$ & 57 & 56 & 76 \\
\hline M34 & $29 *$ & $15^{*}$ & 43 & 58 & $90 *$ \\
\hline
\end{tabular}

*Scores significantly below $(p<.05)$ the ones by controls (CFMT and Famous Faces) or significantly above $(p<.05)$ the ones by controls (CFPT) using Crawford and Howell (1998) procedure.

to recognize throughout the test. For each target individual, participants were presented with a left three-quarters profile view, a front view, and a right three-quarters profile view, each for 3 seconds. After viewing the three images, the participants were presented with three test items in which they needed to identify one of the studied images among two distractors showing different individuals in the same view. This was repeated for all six target faces. In the second phase, test items showed three images (one target and two distractors), and participants had to select the target. Targets in the second phase were always presented in a pose or lighting condition different from the study images. The third phase was similar to the second, but Gaussian noise was added to the images. There were 72 trials (introduction, 18; second phase, 30; third phase, 24). The total number of correct trials for each DP was compared to the results reported by Duchaine and Nakayama (2006). They tested 50 participants with a mean age of 20.2 years $(S D=1.8)$. The mean of total scores was $57.9(S D=7.9)$. (An older group of 13 participants with a mean age of 33.3 years $(S D=6.4)$ scored nearly identically to the college age controls with an average score of $58.9(S D=7.4))$.

\section{Famous faces}

In the Famous Faces Test (Duchaine \& Nakayama, 2005), participants were presented with 60 photographs of famous people, each presented for 3 seconds. Images were cropped to remove hair. Participants were required to name these pictures or provide unique information about the identity of the face (scores in 'Identified' column in Table 2). No feedback was given. Different, though overlapping, famous faces were used for British and American participants. The number of faces identified by British DPs was compared with the results of 22 British participants, with mean age of 25.2 years $(S D=8.1)$; on average controls correctly identified $50.8(S D=6.7)$. Results from American DPs were compared with the results of 19 American participants between the 
ages of 35 and 45; the US controls obtained a mean total score of $52.5(S D=6.6)$. To see whether DPs knew and were exposed to the famous people shown, at the end of the test, participants were read the names of people they did not identify and reported whether they thought they had seen that face many times in daily life. These scores are on the 'Exposed' column in Table 2 and it shows that DPs were exposed to the majority of famous faces presented.

\section{Cambridge face perception test}

In the CFPT (Duchaine, Germine et al., 2007; Duchaine, Yovel et al., 2007), participants were presented with one target face at the top of the screen and six faces below which needed to be sorted according to their similarity to the target face. Each of the six faces was produced by morphing the face of a different individual with the target face. The six faces had different percentages of the target face: $28 \%, 40 \%, 52 \%, 64 \%, 76 \%$, and $88 \%$. Participants had 1 minute for each sort and were encouraged to use the whole minute. An upright and an inverted practice sort preceded the 16 test sorts, 8 of which contained upright faces and 8 inverted faces. For each sort, the deviation of the participant's order from the correct ordering was calculated, and the final score was the sum of the deviations on each sort of a particular orientation. Results from DPs were compared with the results from 11 control participants with the mean age of 33.4 years $(S D=5.5)$. Controls' mean error scores for the upright sorts was $35.5(S D=14.6)$. The mean scores for the inverted sorts was almost twice as high, 63.6 errors $(S D=6.7)$.

\section{Within-category object recognition tests}

Appendix B shows DP's results on object recognition tests using old-new tasks for different categories of objects: cars, horses, houses, tools, scenes, sunglasses and guns. The tests are described in more detail in Duchaine and Nakayama (2005). The procedure was the same for each task. In the study phase, participants saw pictures of 10 objects twice. They were asked to memorize those objects. In the test phase, participants were presented with these pictures and 30 additional distractors and were required to say whether they had seen these objects in the study phase or not. $\mathbf{A}^{\prime}$ and reaction times were computed for each individual on each task. DPs scores were compared with those of 17 graduate students with mean age of 27.8 years (range from 24 to 34). For comparison purposes, the tables in Appendix B also show each DP's score on a similar task using faces. It can be seen that using the same paradigm, all DPs had impaired scores on the face task, but some showed impairments with objects while others performed normally.

\section{Material}

Face versus Non-Face task

Arrays with 25 pictures of animals, objects, food, or landscapes were used $(5 \times 5$ arrays $)$ (inspired by the stimuli used by Lewis \& Edmonds (2003) and Hershler \& Hochstein (2005)). For each face present stimulus, one of the 25 images contained a face. Faces were never repeated. Using Adobe Photoshop, the arrays were converted to greyscale and Gaussian noise (15\%) was added. Arrays with inverted faces were created from the upright arrays. The cell with the face was vertically flipped and the other cells were not changed. Figure 1 shows examples of face present arrays, one with an upright face and 
one with an inverted face. From a viewing distance of $70 \mathrm{~cm}$, the stimuli had a height and width of 12 degrees of visual angle, and the faces had a height and width of 2.5 degrees of visual angle (Figure 1).

Face versus Face Parts task

Sixteen photographs of three-quarter profile views of faces of different individuals with lighting from overhead were transformed to two tone images using Adobe Photoshop. First, each photographic image was blurred in Photoshop, high-pass filtered, then thresholded so that a line drawing of a face was obtained (Figure 2). We placed a face in one of the six tightly packed positions in a visual display. For the face present stimuli, one of the positions would contain an intact face whereas the faces in all other positions were scrambled. This gave the appearance of a face surrounded by a large field of individual features. For the face absent stimuli, faces in all six positions were scrambled. Inverted stimuli with a face present were built from the upright stimuli by vertically flipping the face in the picture while maintaining all other cells in the same orientation. Figure 2 shows examples of face present displays, one with an upright face and one with an inverted face. From a viewing distance of $70 \mathrm{~cm}$, the whole display had a height and width of 7.8 degrees of visual angle, and each individual face had a height and width of 2.6 degrees of visual angle (Figure 2).

\section{Procedure}

Face versus Non-Face task

Blocks consisted of 25 face present displays and 12 face absent displays. Participants were presented with each display for 8 seconds and were instructed to press ' $F$ ' as quickly as possible if a face was present. After pressing ' $\mathrm{F}$ ', the stimulus disappeared and the next trial started. No response was necessary on face absent trials and if participants did not respond the stimulus was removed after 8 seconds. Feedback was given for incorrect responses and for correct responses on absent trials. All participants did an upright block followed by an inverted block. There were five practice trials. The number of hits and false alarms was calculated for each participant in order to compute the measure of sensitivity A' (McMillan \& Creelman, 1990). The mean reaction time for hits was also computed for each participant.

Face versus Face Parts task

The procedure was the same as for the Face versus Non-Face task, except that the number of face present trials was 36. In addition, there were five demonstration trials with face present stimuli at the beginning of each block, before the practice trials, so participants could become familiar with the stimuli.

\section{Results}

\section{Effects of inversion - results from control participants}

Face versus Non-Face task

Left pairs of bars in Figure $3 a$ and $b$ respectively show control accuracy $\left(\mathrm{A}^{\prime}\right)$ and reaction times (RTs) for the Face versus Non-Face task. Differences between upright and 


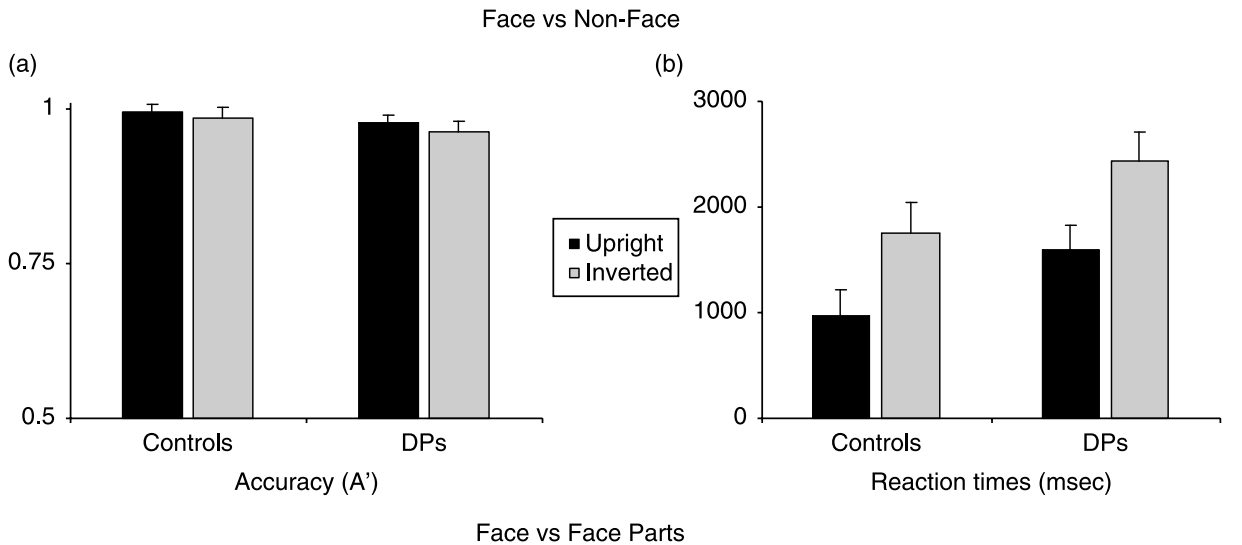

(c)

(d)
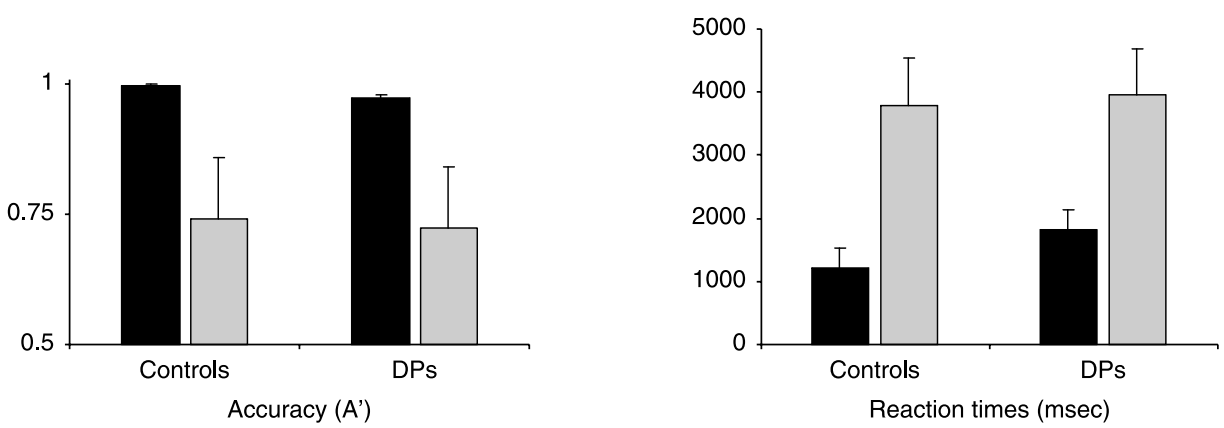

Figure 3. Bar graphs showing mean $A^{\prime}$ and mean reaction times on the detection tasks for control participants and DPs. Error bars represent one standard deviation. a and b show results on the Face versus Non-Face task. Significant inversion effects are seen for reaction times for both controls and DPs. $c$ and $d$ show results on the Face versus Face Parts task. Significant inversion effects are seen for both accuracy and reaction times on the two groups.

inverted were very clear for reaction times but harder to see for accuracy as this measure was very close to ceiling. Controls obtained a mean $\mathrm{A}^{\prime}$ of $0.996(S D=0.011)$ on the upright task, and a mean $\mathrm{A}^{\prime}$ of $0.985(S D=0.017)$ on the inverted block. The mean reaction time was $976 \mathrm{~ms}(S D=235)$ on the upright version and $1752 \mathrm{~ms}(S D=287)$ on the inverted one. Paired $t$ tests were used to compare the inversion effects on both $\mathrm{A}^{\prime}$ and RTs. $\mathrm{A}^{\prime}$ on the upright block were marginally higher than $\mathrm{A}^{\prime}$ on the inverted block $(t(13)=2.15, p<.051)$. Results for reaction times were more dramatic, with significantly slower responses for the inverted condition $(t(13)=-10.87, p<.001)$.

Face versus Face Parts task

Because this test requires the detection of a whole face among distracting face parts, we hoped for an even greater inversion effect than the Face versus Non-Face task. Left pairs of bars in Figure $3 \mathrm{c}$ and d show that orientation indeed had a very strong effect on both accuracy $\left(\mathrm{A}^{\prime}\right)$ and average RT. Controls obtained a mean $\mathrm{A}^{\prime}$ of $0.997(S D=0.004)$ on the upright task and a mean $\mathrm{A}^{\prime}$ of $0.741(S D=0.118)$ on the inverted block. The mean reaction time for hits was $1221 \mathrm{~ms}(S D=314)$ on the upright test and $3784 \mathrm{~ms}$ $(S D=743)$ on the inverted task. Paired $t$ tests were used to compare the inversion 
effects. $\mathrm{A}^{\prime}$ for upright blocks was significantly higher than $\mathrm{A}^{\prime}$ for inverted blocks $(t(13)=8.09, p<.001)$. In addition, RTs on the upright block were significantly faster than RTs on the inverted condition $(t(13)=-15.48, p<.001)$. Thus, in comparison to the Face versus Non-Face task, which showed a strong effect of inversion for RT but a marginal for accuracy, both measures on the Face versus Face Parts task showed very strong inversion effects.

\section{Comparison of DPs and control participants}

The results above demonstrate that face detection can show strong inversion effects, that the task of face detection is significantly more difficult when faces are not in the normal upright configuration. We now describe how developmental prosopagnosics perform on these same detection tasks. Looking ahead, prosopagnosics show the same qualitative pattern of results, performing much better for upright as compared to inverted faces. Yet, there were also some important quantitative differences.

\section{DPs show inversion effects on face detection}

Face versus Non-Face task

Ten DPs were tested with the Face versus Non-Face task. F30b, M30, M32, and M34 did not perform the task or we had a problem with the task when collecting their data. Right pairs of bars in Figure $3 \mathrm{a}$ and $\mathrm{b}$, respectively, show the DPs' $\mathrm{A}^{\prime}$ and RTs for the task comparing upright and inverted performance. Performance was better for upright than inverted but only for the reaction time measure. Accuracy measures were very close to ceiling. They obtained a mean $\mathrm{A}^{\prime}$ of $0.979(S D=0.060)$ on the upright task, and a mean $\mathrm{A}^{\prime}$ of $0.963(S D=0.035)$ on the inverted block. The mean reaction time was 1594 $(S D=821)$ on the upright version and $2429(S D=733)$ on the inverted one. Using paired $t$ tests, $\mathrm{A}^{\prime}$ on the upright block was not significantly different from $\mathrm{A}^{\prime}$ on the inverted block $(t(9)=1.52, n s)$, but RTs were significantly slower on the inverted condition than on the upright one $(t(9)=-8.14, p<.001)$.

Face versus Face Parts task

Right pairs of bars in Figure $3 \mathrm{c}$ and d show that orientation had a more consistent effect on DPs $\mathrm{A}^{\prime}$ and average RTs for the Face versus Face Parts task. They obtained a mean $\mathrm{A}^{\prime}$ of $0.975(S D=0.034)$ on the upright task and a mean $\mathrm{A}^{\prime}$ of $0.724(S D=0.120)$ on the inverted block. The mean reaction time for hits was $1810 \mathrm{~ms}(S D=604)$ on the upright test and $3950 \mathrm{~ms}(S D=798)$ on the inverted task. Using paired $t$ tests, $\mathrm{A}^{\prime}$ for upright blocks was significantly higher than that for inverted blocks $(t(13)=7.72, p<.001)$, and RTs on the upright block were significantly faster than RTs on the inverted condition $(t(13)=-10.83, p<.001)$.

These results show that broadly speaking, prosopagnosic individuals are quite similar to controls in that they both show robust inversion effects on most of the performance measures, with the Face versus Face Parts task showing the greatest effects.

\section{Detailed comparison of normal and prosopagnosic performance}

Not easily evident in summary graphs shown in Figure 3 is the explicit information as to how individual prosopagnosics differ from controls on all measures. In pursuing this goal, 
we must address issues arising from previous reports showing that developmental prosopagnosia is a heterogeneous condition (e.g. Duchaine \& Nakayama, 2005; Harris, Duchaine, \& Nakayama, 2005; Le Grand et al., 2006). Therefore, in addition to evaluating whether there are significant differences between the two groups, it is important to show how individual subjects perform. Moreover, it is also important to be concerned with extraneous reasons why prosopagnosics might perform more poorly, for example, that they might adopt different strategies. Most often cited in evaluated prosopagnosic performance are the possibly contaminating effects of speed accuracy trade offs (Duchaine \& Nakayama, 2005; Gauthier, Behrmann, \& Tarr, 1999).

To deal with these issues, we have plotted in Figure 4 data from all individuals tested so that we can fully appreciate the possible heterogeneity of performance as well as provide an explicit format in evaluating speed accuracy trade offs. For this reason, we have plotted the data of each individual as reaction time versus accuracy. If there are speed accuracy trade offs, there should be a positive trend, with increasing reaction time accompanied by increasing accuracy. The top and bottom rows in Figure 4 show such scatterplots for the Face versus Non-Face task and Face versus Face Parts. Note that there is no evidence for speed accuracy trade offs as we look at the data as a whole. Rather than positive association with accuracy and reaction time (the inverse of speed),

Face versus Non-Face

(a)

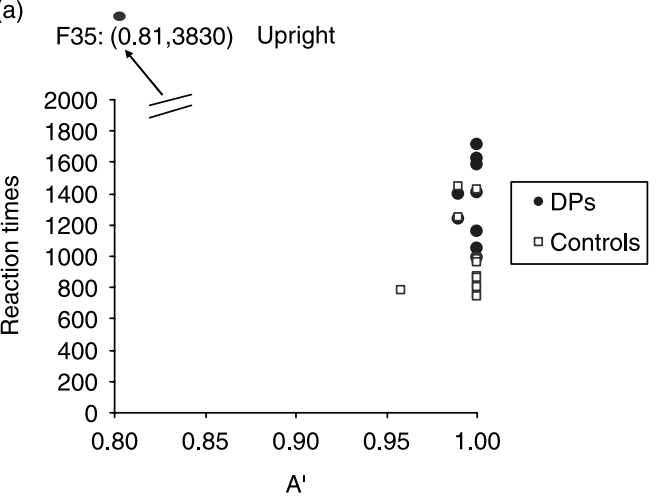

(c)

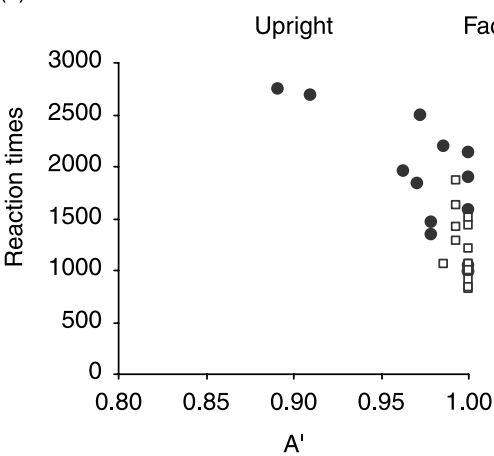

(b)

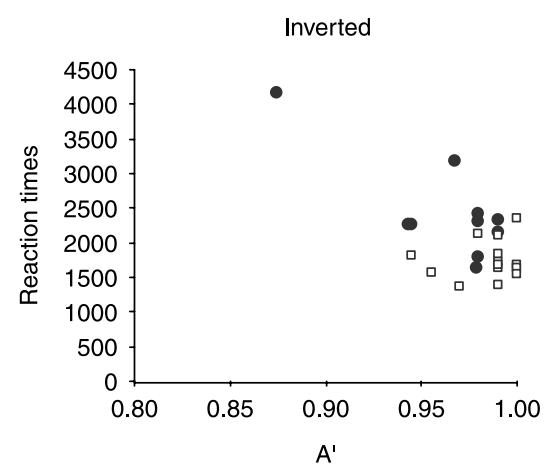

(d)

Figure 4. Reaction time versus accuracy scatterplots showing controls' (open squares) and DPs' (black filled circles) performance on face detection tasks. Note that the scales are different to allow comparison between control and prosopagnosic subjects. 
the plots show either no evidence of trade offs (a and d) or trends in the opposite direction (b and c), especially for the upright Face versus Non-Face task (c). Faster responses are associated with higher accuracy.

Face versus Non-Face task

Figure 4a shows a scatterplot with DPs' and controls' performance on the upright block. Multivariate analysis (MANOVA), which allows us to compare both accuracy and RT measures, was used to contrast the performance of the two groups. There was a significant effect of group $(F(2,21)=7.13, p=.004)$, revealing that DPs were worse than controls on this task. Follow-up one-way independent ANOVAs to investigate the effect of group on each dependent variable showed that there was not a significant effect on $\mathrm{A}^{\prime}(F(1,22)=1.06, p=.315)$ but that there was a significant effect of group on RTs $(F(1,22)=7.22, p=.013)$, with DPs being significantly slower than control participants.

Figure 4b shows a scatterplot with DPs' and controls' performance on the inverted block of the Face versus Non-Face task. MANOVA revealed that there was a significant effect of group $(F(2,21)=4.78, p=.019)$. Subsequent ANOVAs to investigate the effect of group on each dependent variable showed that there was a marginally significant effect on $\mathrm{A}^{\prime}(F(1,22)=4.22, p=.052)$, and a significant effect of group on RTs $(F(1,22)=9.96, p=.005)$. These results show that DPs were significantly slower on this task than control participants.

In addition to the group differences seen in these analyses, we also considered how each DP performed when compared with the control group. Table 3 shows DPs' individual scores on the upright and inverted blocks of the Face versus Non-Face task. Each individual score was compared with scores obtained by controls using Crawford and Howell's (1998) $t$ test. On the upright block, the results from five DPs (F19, F26, F32a, F32b, and F39a) were not significantly different from the ones obtained by controls. The remaining five DPs had significantly slower RTs than control participants and F35 also had a significantly lower $A^{\prime}$ (the result from F35 appears as a clear outlier in the scatterplot in Figure $4 \mathrm{a}$ ). On the inverted block, the results from four DPs (F19, F32a, F32b, F39a) were not significantly different from the ones obtained by controls. Out of the remaining six DPs, five had either significantly higher RTs or lower $A^{\prime}$ than control participants and one DP (F35) was impaired on both measures. Individual hit and false-alarm rates for both upright and inverted blocks can be seen in Appendix C.

Face versus Face Parts task

Figure 4c shows a scatterplot with the performance of the DPs and controls on the inverted block of the Face versus Face Parts Task. MANOVA was used to compare the performance of the two groups considering both dependent variables. There was a significant effect of group $(F(2,25)=5.08, p=.014)$, revealing that DPs performed worse on this task than controls. One-way independent ANOVAs showed that there was a significant effect of group on $\mathrm{A}^{\prime}(F(1,26)=5.60, p=.026)$ and a significant effect of group on RTs $(F(1,26)=10.46, p=.003)$, demonstrating that DPs were significantly slower and less accurate on this task than controls. 
Table 3. DPs' individual results on the Face versus Non-Face task. $A^{\prime}$ and mean reaction times (RTs) for the upright and inverted blocks

\begin{tabular}{lccccc}
\hline & \multicolumn{3}{c}{ Upright } & \multicolumn{2}{c}{ Inverted } \\
\cline { 2 - 3 } Code & $\mathrm{A}^{\prime}$ & $\mathrm{RTs}$ & $\mathrm{A}^{\prime}$ & $\mathrm{RTs}$ \\
\hline F19 & 1.000 & 1160 & 0.980 & 1796 \\
F24 & 1.000 & $1589^{*}$ & 0.980 & $2322^{*}$ \\
F26 & 0.990 & 1235 & $0.944^{*}$ & 2261 \\
F30a & 1.000 & $157 I^{*}$ & & $0.945^{*}$ & 2252 \\
F30b & - & - & - & - \\
F32a & 0.990 & 1393 & 0.980 & 2199 \\
F32b & 1.000 & 1051 & 0.979 & 1636 \\
F35 & $0.807^{*}$ & $3830^{*}$ & & $0.874^{*}$ & $2164^{*}$ \\
F39a & 1.000 & 995 & 0.990 & $2324^{*}$ \\
F39b & 1.000 & $1408^{*}$ & 0.990 & $3188^{*}$ \\
M29 & 1.000 & $1710^{*}$ & 0.967 & - \\
M30 & - & - & - & - \\
M32 & - & - & - & - \\
M34 & - & - & - & \\
\hline
\end{tabular}

*Scores significantly below $(p<.05)$ the ones by controls $\left(A^{\prime}\right)$ or significantly above $(p<.05)$ the ones by controls (RTs) using Crawford and Howell (1998) procedure.

Figure 4d shows a scatterplot with the performance of DPs and controls on the inverted block of the Face versus Face Parts task. Multivariate analysis (MANOVA) revealed that there was not a significant effect of group on this task $(F<0)$.

Table 4 shows DPs' individual scores on the Face versus Face Parts task. Each individual score was compared to control scores. On the upright task, the results for four DPs (F19, F32a, F39a, M32) were within the normal range for both accuracy and RTs. Of the remaining ten DPs, four had one score that was significantly lower than the control scores, and six were impaired on both measures. On the inverted block, M29 was significantly slower than controls on this task. All other results were within the normal range. Individual hit and false-alarm rates for both upright and inverted blocks can be seen in Appendix C.

In summary, it is clear that DPs performance is worse than controls in detecting faces. This difference is evident for reaction times in the two tasks, and for both accuracy and reaction times in the more difficult Face versus Face Parts Task. Note, however, that some individual DPs had performance within the normal range on both tasks.

The Face versus Face Parts task caused robust inversion effects for both controls and DPs and differentiated mean performance between the two groups. A more systematic view of these results can be best illustrated in a summary graph where an additional bootstrap resampling procedure was used (Davison \& Hinkley, 1997; Efron \& Tibshirani, 1993). Using the original data for each group on upright and inverted blocks (as shown in Figure 4c and d), bootstrapped datasets with 14 observations (same size as the original sample in each condition) were resampled with replacement. The mean of each bootstrapped sample was calculated and plotted on Figure 5, with the $x$-axis showing accuracy and the $y$-axis showing reaction times. The procedure was repeated for 200 samples. Figure 5 clearly shows the separate bootstrap distributions of upright and 
132 Lucia Garrido et al.

Table 4. DPs' individual results on the Face versus Face Parts task. $A^{\prime}$ and mean reaction times (RTs) for the upright and inverted blocks

\begin{tabular}{|c|c|c|c|c|}
\hline \multirow[b]{2}{*}{ Code } & \multicolumn{2}{|c|}{ Upright } & \multicolumn{2}{|c|}{ Inverted } \\
\hline & $A^{\prime}$ & RTs & $A^{\prime}$ & RTs \\
\hline FI9 & 1.000 & 1047 & 0.550 & 2757 \\
\hline F24 & $0.972^{*}$ & $2492 *$ & 0.600 & 4466 \\
\hline F26 & $0.979 *$ & 1345 & 0.750 & 3955 \\
\hline $\mathrm{F} 30 \mathrm{a}$ & $0.979 *$ & 1458 & 0.646 & 4010 \\
\hline $\mathrm{F} 30 \mathrm{~b}$ & $0.97 I^{*}$ & $|83| *$ & 0.600 & 3835 \\
\hline $\mathrm{F} 32 \mathrm{a}$ & 1.000 & 991 & 0.913 & 3862 \\
\hline $\mathrm{F} 32 \mathrm{~b}$ & 1.000 & $2129 *$ & 0.824 & 2394 \\
\hline F35 & $0.891 *$ & $2754 *$ & 0.724 & 5009 \\
\hline F39a & 1.000 & 979 & 0.881 & 3764 \\
\hline F39b & 1.000 & $1896^{*}$ & 0.795 & 3555 \\
\hline M29 & $0.986^{*}$ & $2188^{*}$ & 0.534 & $5566 *$ \\
\hline M30 & $0.963^{*}$ & $1960 *$ & 0.765 & 3884 \\
\hline M32 & 1.000 & 1584 & 0.792 & 3839 \\
\hline M34 & $0.910 *$ & $2689 *$ & 0.768 & 4400 \\
\hline
\end{tabular}

*Scores significantly below $(p<.05)$ the ones by controls $\left(\mathrm{A}^{\prime}\right)$ or significantly above $(p<.05)$ the ones by controls (RTs) using Crawford and Howell (1998) procedure.

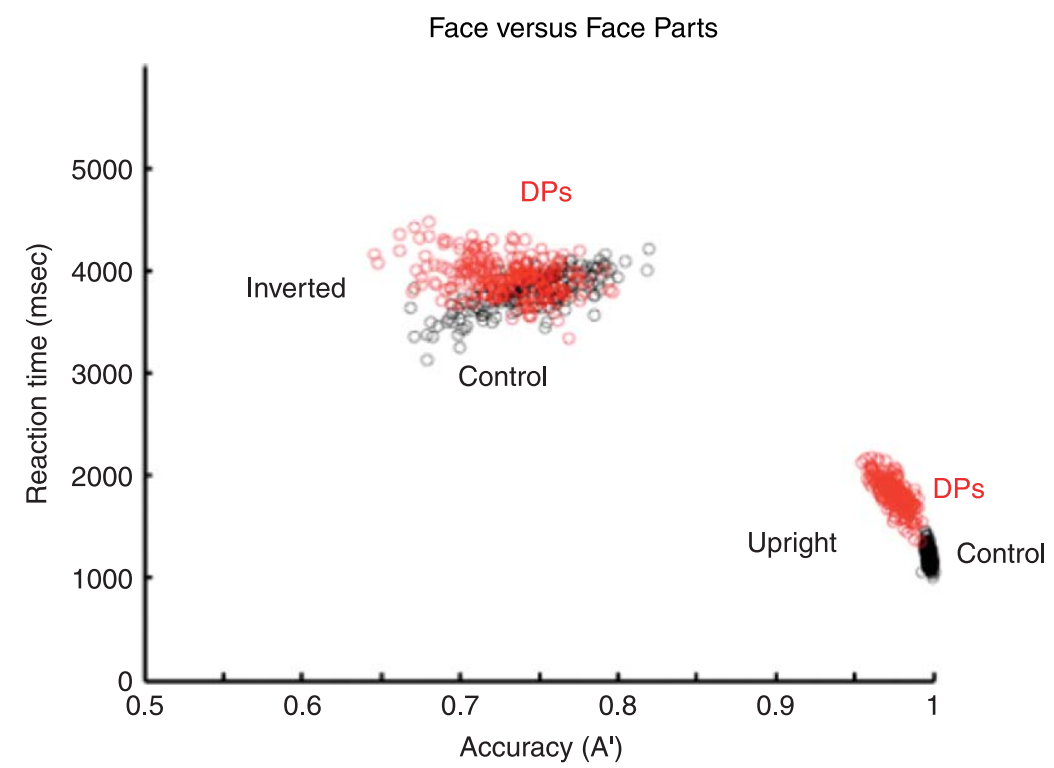

Figure 5. Bootstrapped means $(N=200)$ of control subjects (black) and DPs (red) on the Face versus Face Parts task. Note the separate distributions for upright and inverted results that denote the robust inversion effects for both accuracy and reaction times on this task. Note also the separate distributions for controls and DPs results on the upright block. 
inverted results and a distinction between bootstrapped means of control participants (black) and DPs (red) on the upright block of the task. This plot makes it clear that there is little difference between the distributions of the inverted scores. Thus, prosopagnosic subjects are far worse for the detection of upright Faces versus Face Parts, but are nearly comparable to normals for the detection of inverted faces.

\section{Discussion}

\section{Inversion effects}

We have found inversion effects in both of our search tasks. For the Face versus NonFace this was clearly evident in reaction times and for the Face versus Face Parts, it was clearly evident for both reaction times and accuracy. This indicates that we cannot place the process of face detection in a different category, that detection like recognition clearly shows the characteristic so closely associated with face processing.

To show the consistency of the inversion effect for both controls and prosopagnosics, we plotted all individual data for reaction times. Figure 6 shows inverted versus upright reaction times for all subjects, control and prosopagnosic, for the two tasks. The shallow solid diagonal line represents a slope of unity and the steeper line represents a slope of 2:1. Note that with the exception of one outlier prosopagnosic in the Face versus Non-Face data, all data points conform to an approximately 2:1 slope, thus inverted reaction times are approximately twice as large as upright ones (Figure 6a). This is true for both normal and prosopagnosic individuals. The inversion effect is even greater for the Face versus Face Parts task. For the inverted case, many individuals had to have the inverted face pointed out to them before doing the task.

These results are inconsistent with previous findings by Lewis and Edmonds (2003, 2005), Rousselet et al. (2003), and VanRullen (2006) who found small effects of inversion on their face detection tasks. The paradigm we used was not very different from the ones used in the two first studies. However, they used natural scenes whereas the Face versus Non-Face task used greyscale photographs degraded with noise and the

(a)

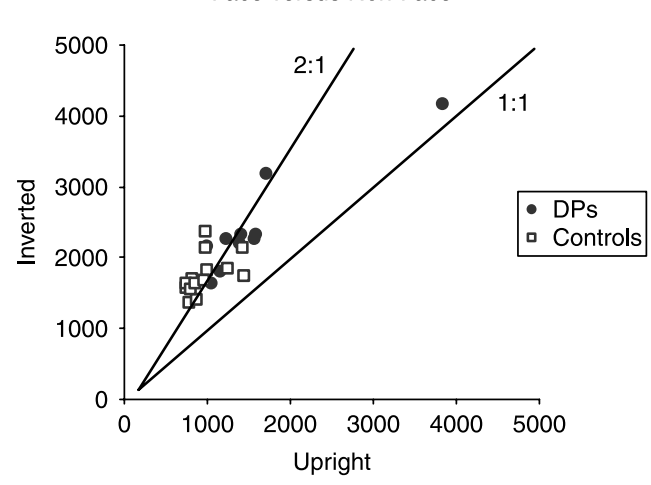

(b)

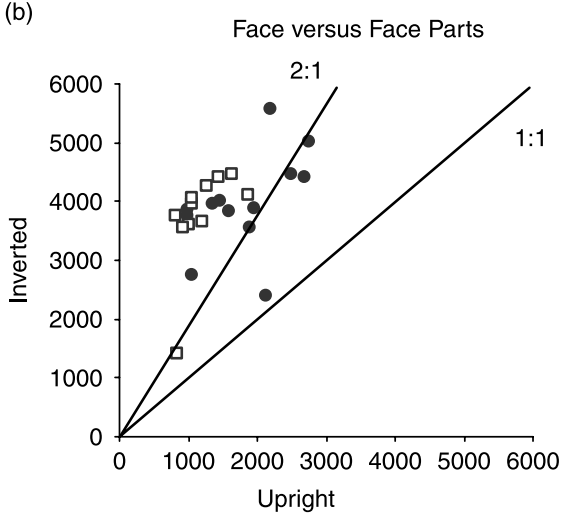

Figure 6. Scatterplots showing inversion effects on reaction times for the Face versus Non-Face task (a) and Face versus Face Parts (b). The steep diagonal line has a slope of two (labelled 2:I) while the shallow line has a slope of unity $(I: I)$. Results above the unity line show that participants were faster for upright than inverted trials, and results above the 2:I line show that participants were about two times faster on the upright block than on the inverted block. 
Face versus Face Parts test used two-tone faces. Hence, cues that are present in their stimuli but not in ours appear to be especially important for inverted face detection. We believe that the presence of contextual cues, external facial features and skin colour in those previous studies might have led to small inversion effects. Our stimuli, though, were more reliant on the structure of the face. In the Face versus Non-Face, we removed external features such as the body, and pictures were greyscale so skin colour could not facilitate judgements. The Face versus Face Parts, on the other hand, relied on the configuration of the face among competing face parts.

Our large inversion effects are consistent with studies using Mooney stimuli (Andrews \& Schluppeck, 2004; George et al., 1997, 2005; Kanwisher et al., 1998). Mooney stimuli are also two-tone images as the pictures used in one of our tasks, hence it may be that there is something particular about this type of stimuli that causes robust inversion effects. However, our tasks depart from the usual paradigms in which Mooney stimuli have been used, which consist of classification tasks using centrally presented stimuli. We employed visual search-type tasks in which the faces were surrounded by distractors because these tasks simulate better face detection in everyday life.

These results are also consistent with the many other inversion effects seen in face processing for tasks requiring perceptual or structural encoding (e.g. Freire et al., 2000; Riesenhuber et al., 2004; Yovel \& Kanwisher, 2004) or memory (e.g. Diamond \& Carey, 1986; Duchaine \& Nakayama, 2006; Moscovitch \& Moscovitch, 2000; Yin, 1969). Our results therefore demonstrate that the face detection, like the other face processes, is also handled by mechanisms tuned to the upright orientation. Our results, though, do not support previous claims that detection is a separate stage from recognition because it is not impeded by the inversion of faces (Lewis \& Edmonds, 2003, 2005). Note, however, that even though some face detection experiments show clear inversion effects, we believe that it is still likely that detection and recognition are separable. It may be that face detection is strongly affected by inversion (a distinguishing characteristic of all face processing) and yet could still be a separate mechanism from other face processing.

\section{Results with DPs}

Results with the DPs showed that most of them have some difficulties on face detection tasks. This is particularly evident in looking at the raw data for all individuals (normals and DPs) on the Face versus Face Parts test shown in Figure 4c, and at the bootstrapped distributions in Figure 5. This finding contrasts with previous studies in which it was shown that the DPs studied did not have problems with face detection (de Gelder \& Rouw, 2000; Duchaine et al., 2003, 2006; Le Grand et al., 2006). Given the heterogeneity seen in DP (Duchaine \& Nakayama, 2005; Harris et al., 2005; Le Grand et al., 2006), it is possible that none of the cases studied before did have face detection impairments. However, most of these studies required face categorization (comparing faces with objects or scrambled faces) which is very easy and does not closely simulate face detection in everyday life.

DPs' impairments were observed on both the upright and the inverted blocks of the Face versus Non-Face task, and on the upright block of the Face versus Face Parts. We think that DPs were more likely to have impaired scores on the upright blocks than on the inverted ones because the first involves face-specific processes whereas the processing of inverted faces might also rely on different, non-face, mechanisms (Moscovitch \& Moscovitch, 2000). In addition, we suspect that there were more 
impaired scores for DPs on the inverted block of the Face versus Non-Face than on the Face versus Face Parts task because controls' scores on the Face versus Face Parts task were too variable.

One possible explanation for the impairments in face detection could be that they were caused by low-level perceptual impairments. DPs' results however do not support this interpretation. All 12 DPs tested on the BORB performed within the normal range on low-level perceptual tasks from that battery. Another possible interpretation for the observed detection impairments could be that the DPs with additional problems with object recognition, given their more widespread visual difficulties, would be the ones showing the worst performance on detection tasks. However, our results do not seem to support this interpretation either. Two DPs who had problems with face detection showed normal performance on object tests (F30a, M30). Both these DPs had accuracy within the normal range on all tests of object recognition (see Appendix B). They both had one task in which they were significantly slower than controls, but we do not consider these results to be sufficient to consider them as having impaired object recognition. Conversely, all DPs who did not have any difficulties on the face detection tasks (F19, F32a, F39a, M32) showed at least two impaired scores on the object recognition tests.

Three other criticisms can be raised regarding the conclusion that these results show that these DPs have problems detecting faces. First, it could be that they were just more cautious when performing the tasks so they responded more slowly. Although this might be true for some cases, other DPs showed both impaired accuracy and impaired response times. Second, it could be that slower reaction times on the Face versus Non-Face task were caused by DPs' difficulties processing the distractor objects, particularly since some of these DPs also have impairments with objects. However, some of these individuals performed normally on within-category object recognition tests. More importantly, object recognition difficulties would not be expected to impair performance on the Face versus Face Parts test, and the DPs did more poorly on this task than the Face versus Non-Face task. A third criticism could be that the stimulus presentation times were too long. de Gelder and Rouw (2000) described very interesting results showing that an acquired prosopagnosic's performance on a face categorization task was normal when stimuli were briefly presented, but severely impaired when presentation times were unlimited. The authors suggested that his poor performance with unlimited presentations could result from interference from his damaged face recognition system. The present data do not address this possibility and future studies could present stimuli for very brief presentation times to address it.

The impaired face detection shown by some DPs is compatible with the developmental proposal by Johnson (2005) and de Gelder and Stekelenburg (2005) that some cases of developmental prosopagnosia might originate in a deficit in the mechanism carrying out face detection. They proposed that this system is subcortical and its input is crucial for the normal development of cortical specialized face processing. Note, however, that many of our DPs with impaired face detection also have object recognition impairments and this developmental account does not provide an obvious explanation for their object recognition deficits. The combination of face and object problems suggests that these deficits originate in the neighbouring cortical areas responsible for face and object processing rather than being a downstream developmental effect of deficits in subcortical mechanisms. Recent evidence from microstimulation in primates also suggests a crucial role of face-specific cortical regions in face detection (Afraz, Kiani, \& Esteky, 2006). In this study, when face-selective 
neurons in the inferotemporal cortex were stimulated, monkeys were more likely to classify a noisy visual stimulus as a face.

Crucially, although most of the DPs showed impairments with face detection, three DPs (F19, F32a, F39a) performed normally on both tests. A fourth DP (M32) also showed normal scores on the Face versus Face Parts but was not tested with the Face versus Non-Face task. Their normal face detection coupled with severely impaired face recognition suggests that face detection and face recognition are carried out by different mechanisms. It could be that these four DPs were simply less impaired with face recognition than the other DPs, but inspection of their scores on the CFMT and the Famous Faces Test show that their impairments were comparable to the other DPs.

On the other hand, none of these four DPs had impairments on the CFPT which was designed to assess perceptual processes, rather than memory ones. Note though that other DPs also did not show impairments on this task but did show impaired performance on the Face versus Non-Face and the Face versus Face Parts task. It will be interesting to analyse in the future whether face detection impairments can be dissociated from structural encoding impairments. At the moment, even though impaired scores in the CFPT clearly demonstrate problems in perceptual encoding, we do not feel that normal scores provide definitive evidence that these mechanisms function normally. Therefore, future studies need to include a larger number of perceptual tasks to address this issue.

To conclude, the present study showed that finding a face in a visual scene is robustly affected by inversion. Control participants were much slower and much less accurate when faces were upside-down in two tasks of face detection. These results show that face detection, like other face processing tasks, is strongly affected by inversion. DPs also showed significant inversion effects. In addition, most DPs tested showed slower performance or made more errors on the two tasks of face detection. The group differences were more clearly seen for the Face versus Face Parts task, which was more difficult than the Face versus Non-Face task and highly reliant on configural processing. But, crucially, four individuals did not show any impairment. Their normal performance suggests that face detection involves mechanisms separate from those used for recognition and that developmental prosopagnosia can result from impairments at different mechanisms of face processing. The variability in face detection performance in our DPs adds to the already considerable evidence demonstrating that DP is a heterogeneous condition (Duchaine \& Nakayama, 2005; Harris et al., 2005; Kress \& Daum, 2003; Le Grand et al., 2006).

\section{Acknowledgements}

We are extremely grateful to all DPs for their time and effort. We thank Laura Germine, Anna Grossetete, George Gordon and Carl Mueller for their assistance. This work was supported by an ESRC grant (RES-061-23-0400) to B.D., and NIH grant to K.N. (R01 EY13602) and a studentship from the Foundation for Science and Technology, Portugal, to L.G.

\section{References}

Afraz, S., Kiani, R., \& Esteky, H. (2006). Microstimulation of inferotemporal cortex influences face categorization. Nature, 442, 692-695.

Andrews, T., \& Schluppeck, D. (2004). Neural responses to Mooney images reveal a modular representation of faces in human visual cortex. Neuroimage, 21, 91-98. 
Bruce, V., \& Young, A. (1986). Understanding face recognition. British Journal of Psychology, 77(Pt 3), 305-327.

Bruyer, R., Laterre, C., Seron, X., Feyereisen, P., Strypstein, E., Pierrard, E., et al. (1983). A case of prosopagnosia with some preserved covert remembrance of familiar faces. Brain and Cognition, 2, 257-284.

Burton, A. M., Bruce, V., \& Hancock, P. J. B. (1999). From pixels to people: A model of familiar face recognition. Cognitive Science, 23, 1-31.

Crawford, J. R., \& Howell, D. C. (1998). Comparing an individual's test score against norms derived from small samples. Clinical Neuropsychologist, 12, 482-486.

Davison, A., \& Hinkley, D. (1997). Bootsrap methods and their application. Cambridge: Cambridge University Press.

de Gelder, B., \& Rouw, R. (2000). Configural face processes in acquired and developmental prosopagnosia: Evidence for two separate face systems? Neuroreport, 11, 3145-3150.

de Gelder, B., \& Stekelenburg, J. J. (2005). Naso-temporal asymmetry of the N170 for processing faces in normal viewers but not in developmental prosopagnosia. Neuroscience Letters, $376,40-45$.

Diamond, R., \& Carey, S. (1986). Why faces are and are not special: An effect of expertise. Journal of Experimental Psychology: General, 115, 107-117.

Duchaine, B., Germine, L., \& Nakayama, K. (2007). Family resemblance: Ten family members with prosopagnosia and within-class object agnosia. Cognitive Neuropsychology, 24(4), 419-430.

Duchaine, B., \& Nakayama, K. (2005). Dissociations of face and object recognition in developmental prosopagnosia. Journal of Cognitve Neuroscience, 17, 249-261.

Duchaine, B., \& Nakayama, K. (2006). The Cambridge face memory test: Results for neurologically intact individuals and an investigation of its validity using inverted face stimuli and prosopagnosic participants. Neuropsychologia, 44, 576-585.

Duchaine, B. C., Nieminen-von, W. T., New, J., \& Kulomaki, T. (2003). Dissociations of visual recognition in a developmental agnosic: Evidence for separate developmental processes. Neurocase, 9, 380-389.

Duchaine, B. C., Yovel, G., Butterworth, B., \& Nakayama, K. (2006). Prosopagnosia as an impairment to face-specific mechanisms: Elimination of the alternative hypotheses in a developmental case. Cognitive Neuropsychology, 23, 714-747.

Duchaine, B., Yovel, G., \& Nakayama, K. (2007). No global processing deficit in the Navon task in 14 developmental prosopagnosics. Social Cognitive and Affective Neuroscience, 2, 104-113.

Efron, B., \& Tibshirani, R. (1993). An introduction to the bootstrap. New York: Chapman and Hall.

Farah, M., Tanaka, J., \& Drain, H. (1995). What causes the face inversion effect? Journal of Experimental Psychology: Human Perception and Performance, 21, 628-634.

Freire, A., Lee, K., \& Symons, L. (2000). The face inversion effect as a deficit in the encoding of configural information: Direct evidence. Perception, 29, 159-170.

Gauthier, I., Behrmann, M., \& Tarr, M. (1999). Can face recognition really be dissociated from object recognition? Journal of Cognitive Neuropsychology, 11, 349-370.

George, N., Jemel, B., Fiori, N., Chaby, L., \& Renault, B. (2005). Electrophsyiological correlates of facial decision: Insights from upright and upside-down Mooney-face perception. Cognitive Brain Research, 24, 663-673.

George, N., Jemel, B., Fiori, N., \& Renault, B. (1997). Face and shape repetition effects in humans: A spatio-temporal ERP study. Neuroreport, 8, 1417-1423.

Harris, A. M., Duchaine, B. C., \& Nakayama, K. (2005). Normal and abnormal face selectivity of the M170 response in developmental prosopagnosics. Neuropsychologia, 43, 2125-2136.

Haxby, J. V., Hoffman, E. A., \& Gobbini, M. I. (2000). The distributed human neural system for face perception. Trends in Cognitive Sciences, 4, 223-233.

Hershler, O., \& Hochstein, S. (2005). At first sight: A high-level pop out effect for faces. Vision Research, 45, 1707-1724.

Johnson, M. H. (2005). Subcortical face processing. Nature Reviews Neuroscience, 6, 766-774. 
Kanwisher, N., Tong, F, \& Nakayama, K. (1998). The effect of inversion on the human fusiform face area. Cognition, 68, B1-B11.

Kress, T., \& Daum, I. (2003). Developmental prosopagnosia: A review. Behavioural Neurology, 14, 109-121.

Le Grand, R., Cooper, P. A., Mondloch, C. J., Lewis, T. L., Sagiv, N., de, G. B., et al. (2006). What aspects of face processing are impaired in developmental prosopagnosia? Brain and Cognition, 61, 139-158.

Lewis, M. B., \& Edmonds, A. J. (2003). Face detection: Mapping human performance. Perception, 12, 1309-1336.

Lewis, M. B., \& Edmonds, A. J. (2005). Searching for faces in scrambled scenes. Visual Cognition, 32, 903-920.

Lewis, M. B., \& Ellis, H. D. (2003). How we detect a face: A survey of psychological evidence. International Journal of Imaging Systems and Technology, 13, 3-7.

McKone, E., Kanwisher, N., \& Duchaine, B. (2007). Can generic expertise explain special processing for faces? Trends in Cognitive Sciences, 11(1), 8-15.

McMillan, N., \& Creelman, C. (1990). Response bias: Characteristics of detection theory, threshold theory and 'nonparametric' indexes. Psychological Bulletin, 107(3), 401-413.

Moscovitch, M., \& Moscovitch, D. (2000). Super face-inversion effects for isolated internal or external features, and for fractured faces. Cognitive Neuropsychology, 17, 201-219.

Purcell, D. G., \& Stewart, A. L. (1986). The face-detection effect. Bulletin of the Psychonomic Society, 24, 118-120.

Purcell, D. G., \& Stewart, A. L. (1988). The face-detection effect - configuration enhances detection. Perception and Psychophysics, 43, 355-366.

Riddoch, M., \& Humphreys, G. (1993). BORB: Birmingham object recognition battery. Hove, UK: Erlbaum.

Riesenhuber, M., Jarudi, I., Gilad, S., \& Sinha, P. (2004). Face processing in humans is compatible with a shape-based model of vision. Proceedings of the Royal Society B, 271, S448-S450.

Rossion, B., Caldara, R., Seghier, M., Schuller, A. M., Lazeyras, F., \& Mayer, E. (2003). A network of occipito-temporal face-sensitive areas besides the right middle fusiform gyrus is necessary for normal face processing. Brain, 126, 2381-2395.

Rousselet, G. A., Mace, M. J. M., \& Fabre-Thorpe, M. (2003). Is it an animal? Is it a human face? Fast processing in upright and inverted natural scenes. Journal of Vision, 3, 440-455.

Schiltz, C., Sorger, B., Caldara, R., Ahmed, F., Mayer, E., Goebel, R., et al. (2006). Impaired face discrimination in acquired prosopagnosia is associated with abnormal response to individual faces in the right middle fusiform gyrus. Cerebral Cortex, 16, 574-586.

VanRullen, R. (2006). On second glance: Still no high-level pop-out effect for faces. Vision Research, 46, 3017-3027.

Viola, P., \& Jones, M. J. (2004). Robust real-time face detection. International Journal of Computer Vision, 57(2), 137-154.

Yang, M. H., Kriegman, D. J., \& Ahuja, N. (2002). Detecting faces in images: A survey. IEEE Transactions on Pattern Analysis and Machine Intelligence, 24, 34-58.

Yin, R. K. (1969). Looking at upside-down faces. Journal of Experimental Psychology, 81, 141-145.

Yovel, G., \& Kanwisher, N. (2004). Face perception: Domain specific, not process specific. Neuron, 44, 889-898.

Received 24 May 2007; revised version received 17 September 2007 


\section{Appendix A}

Performance on four tasks from BORB

\begin{tabular}{lcccc}
\hline & \multicolumn{3}{c}{ BORB } \\
\cline { 2 - 5 } & Length match & Size match & Orientation match & Position of gap \\
\cline { 2 - 5 } Controls $^{\mathrm{a}}$ & $26.9(1.6)$ & $27.3(2.4)$ & $24.8(2.6)$ & $35.1(4.0)$ \\
\hline F19 & - & - & - & - \\
F24 & 27 & 29 & 22 & 35 \\
F26 & 28 & 28 & 26 & 35 \\
F30a & 27 & 26 & 29 & 36 \\
F30b & 26 & 27 & 27 & 39 \\
F32a & 27 & 25 & 26 & 36 \\
F32b & 30 & 26 & 26 & 35 \\
F35 & 30 & 29 & 26 & 36 \\
F39a & 29 & 27 & 27 & 38 \\
F39b & 29 & 26 & 28 & 38 \\
M29 & 26 & 27 & 27 & 39 \\
M30 & 26 & 29 & 30 & - \\
M32 & 27 & 29 & 24 & - \\
M34 & - & - & - & \\
\hline
\end{tabular}

${ }^{a}$ Controls' results for BORB correspond to the published norms (Riddoch \& Humphreys, 1993). Values in the table correspond to means and standard deviations (between parentheses).

\section{Appendix B}

a) Performance on object recognition tests - accuracy $\left(A^{\prime}\right)$

\begin{tabular}{|c|c|c|c|c|c|c|c|c|}
\hline & Cars & Horses & Houses & Tools & Scenes & Sunglasses & Guns & Faces \\
\hline \multicolumn{9}{|c|}{ Controls } \\
\hline$M$ & 0.94 & 0.95 & 0.97 & 0.96 & 0.97 & 0.90 & 0.92 & 0.96 \\
\hline$S D$ & 0.04 & 0.03 & 0.03 & 0.02 & 0.03 & 0.05 & 0.04 & 0.02 \\
\hline FI9 & 0.91 & 0.95 & 0.99 & 0.95 & 0.99 & 0.98 & 0.90 & $0.69 *$ \\
\hline F24 & $0.8 I^{*}$ & 0.94 & 0.94 & 0.97 & 0.99 & 0.95 & 0.94 & $0.83 *$ \\
\hline F26 & $0.74^{*}$ & $0.74 *$ & 0.93 & $0.82 *$ & 0.93 & 0.83 & $0.78^{*}$ & $0.86 *$ \\
\hline $\mathrm{F} 30 \mathrm{a}$ & 0.87 & 0.92 & 0.93 & 0.95 & 0.93 & 0.82 & 0.87 & $0.89 *$ \\
\hline F30b & 0.87 & 0.93 & 0.96 & - & - & - & - & $0.86 *$ \\
\hline $\mathrm{F} 32 \mathrm{a}$ & $0.86^{*}$ & 0.93 & 0.98 & 0.98 & 1.00 & 0.92 & 0.88 & $0.83 *$ \\
\hline$F 32 b$ & $0.86 *$ & 0.96 & 0.94 & - & - & - & - & 0.93 \\
\hline F35 & 0.88 & 0.91 & $0.85^{*}$ & $0.87^{*}$ & 0.92 & 0.85 & $0.73 *$ & $0.78^{*}$ \\
\hline F39a & $0.78^{*}$ & 0.92 & 0.93 & $0.87^{*}$ & 0.98 & 0.83 & $0.72 *$ & $0.90 *$ \\
\hline F39b & $0.69 *$ & $0.87 *$ & 0.94 & $0.89 *$ & 0.93 & 0.81 & $0.77^{*}$ & $0.66 *$ \\
\hline M29 & 0.91 & 0.91 & 0.96 & 0.98 & 0.95 & 0.90 & 0.94 & 0.93 \\
\hline M30 & 0.90 & 0.91 & 0.95 & 0.99 & 1.00 & 0.95 & 0.91 & $0.83 *$ \\
\hline M32 & 0.98 & 0.92 & $0.91 *$ & 1.00 & 1.00 & 0.98 & 0.96 & $0.88 *$ \\
\hline M34 & $0.84^{*}$ & - & $0.86 *$ & - & $0.91 *$ & - & 0.85 & $0.79 *$ \\
\hline
\end{tabular}

*Scores significantly below $(p<.05)$ the ones by controls using Crawford and Howell (1998) procedure. 
140 Lucia Garrido et al.

b) Performance on object recognition tests - reaction times (milliseconds)

\begin{tabular}{lcccccccc}
\hline & Cars & Horses & Houses & Tools & Scenes & Sunglasses & Guns & Faces \\
\hline Controls & & & & & & & & \\
M & 1205 & 1186 & 998 & 963 & 896 & 957 & 1189 & 988 \\
SD & 427 & 284 & 197 & 250 & 186 & 144 & 278 & 217 \\
\hline FI9 & 1607 & 1401 & $1428^{*}$ & 1056 & 1048 & $1402^{*}$ & 1307 & $1717^{*}$ \\
F24 & 1949 & $2012^{*}$ & $1711^{*}$ & 1211 & 1172 & $1450^{*}$ & 1602 & $2183^{*}$ \\
F26 & $1977^{*}$ & 1430 & $1793^{*}$ & $1616^{*}$ & $1430^{*}$ & $132 I^{*}$ & 1603 & $2122^{*}$ \\
F30a & 1272 & 1353 & $1545^{*}$ & 1203 & 1221 & 1140 & 1099 & $1952^{*}$ \\
F30b & 1426 & 1268 & $1492^{*}$ & - & - & - & - & $1763^{*}$ \\
F32a & 1781 & 1627 & $1420^{*}$ & 1400 & 1110 & 1140 & 1402 & $2199^{*}$ \\
F32b & 1371 & 1111 & $1667^{*}$ & - & - & - & - & $1434^{*}$ \\
F35 & $2111^{*}$ & $1894^{*}$ & $2159^{*}$ & $1502^{*}$ & $1791^{*}$ & $192 I^{*}$ & $205 I^{*}$ & $2352^{*}$ \\
F39a & 1088 & 1027 & 1168 & 1241 & 1066 & $132 I^{*}$ & 1238 & $148 I^{*}$ \\
F39b & 1400 & 1020 & 1110 & 867 & 1231 & 1108 & 1287 & $161 I^{*}$ \\
M29 & 1898 & 1683 & $166 I^{*}$ & $1440^{*}$ & $1735^{*}$ & $2457^{*}$ & $2365^{*}$ & $1497^{*}$ \\
M30 & 1481 & 1697 & 1194 & 1116 & 938 & $1288^{*}$ & 1351 & $2306^{*}$ \\
M32 & 1405 & $2374^{*}$ & $1845^{*}$ & $1697^{*}$ & 1209 & $2112^{*}$ & $2158^{*}$ & $2750^{*}$ \\
M34 & 1933 & - & $1679^{*}$ & - & $1302^{*}$ & - & 1643 & $198 I^{*}$ \\
\hline
\end{tabular}

*Scores significantly above $(p<.05)$ the ones by controls using Crawford and Howell (1998) procedure.

\section{Appendix C}

Hit rate and false-alarm rate on face detection tasks. Misses correspond to errors on face present trials ( 25 on Face versus Non-Face and 36 on Face versus Face Parts). False alarms correspond to errors on face absent trials ( 12 for both tasks).

\begin{tabular}{|c|c|c|c|c|c|c|c|c|}
\hline \multirow[b]{3}{*}{ Code } & \multicolumn{4}{|c|}{ Face versus Non-Face } & \multicolumn{4}{|c|}{ Face versus Face Parts } \\
\hline & \multicolumn{2}{|c|}{ Upright } & \multicolumn{2}{|c|}{ Inverted } & \multicolumn{2}{|c|}{ Upright } & \multicolumn{2}{|c|}{ Inverted } \\
\hline & Hit rate & $\begin{array}{l}\text { False-alarm } \\
\text { rate }\end{array}$ & Hit rate & $\begin{array}{l}\text { False-alarm } \\
\text { rate }\end{array}$ & Hit rate & $\begin{array}{l}\text { False-alarm } \\
\text { rate }\end{array}$ & Hit rate & $\begin{array}{l}\text { False-alarm } \\
\text { rate }\end{array}$ \\
\hline FI9 & 100 & 0 & 92 & 0 & 100 & 0 & 86 & 83 \\
\hline F24 & 100 & 0 & 92 & 0 & 89 & 0 & 53 & 42 \\
\hline F26 & 96 & 0 & 88 & 8 & 100 & 8 & 67 & 33 \\
\hline $\mathrm{F} 30 \mathrm{a}$ & 100 & 0 & 96 & 17 & 100 & 8 & 50 & 33 \\
\hline $\mathrm{F} 30 \mathrm{~b}$ & - & - & - & - & 97 & 8 & 53 & 42 \\
\hline $\mathrm{F} 32 \mathrm{a}$ & 96 & 0 & 92 & 0 & 100 & 0 & 78 & 8 \\
\hline $\mathrm{F} 32 \mathrm{~b}$ & 100 & 0 & 100 & 8 & 100 & 0 & 81 & 33 \\
\hline F35 & 84 & 42 & 96 & 42 & 94 & 33 & 53 & 25 \\
\hline F39a & 100 & 0 & 96 & 0 & 100 & 0 & 86 & 25 \\
\hline $\mathrm{F} 39 \mathrm{~b}$ & 100 & 0 & 96 & 0 & 100 & 0 & 67 & 25 \\
\hline M29 & 100 & 0 & 96 & 8 & 94 & 0 & 28 & 25 \\
\hline M30 & - & - & - & - & 94 & 8 & 69 & 33 \\
\hline M32 & - & - & - & - & 100 & 0 & 56 & 17 \\
\hline M34 & - & - & - & - & 86 & 17 & 36 & 8 \\
\hline
\end{tabular}

